\title{
$\infty$ \\ A Nova Definição e Estadiamento Universal da InsuficiênCIa CARdíaCA
}

\begin{abstract}
Autores
Ana Paula Chedid Mendes ${ }^{1}$, Evandro Tinoco Mesquita ${ }^{2}$ ${ }^{1}$ Universidade Federal Fluminense; Sexta Enfermaria Santa Casa Rio de Janeiro

${ }^{2}$ Presidente do DEIC; Vice Presidente da Sociedade Interamericana de Cardiologia; Professor da Universidade Federal Fluminense; Educador CTEB-UHG; Instituto Cardiovascular CHN/DASA; Sexta Enfermaria Santa Casa Rio de Janeiro; Sexta Enfermaria Santa Casa Rio de Janeiro
\end{abstract}

A primeira definição de insuficiência cardíaca (IC) publicada em livro foi de Lewis ${ }^{1}$, em 1933, e apresentava forte correlação com estado edematoso, uma vez que a IC, na época, era o principal fator fisiopatológico contribuinte para o edema e dispnéia. Era descrita, portanto, como "condição em que o coraçáo náo consegue descarregar seu conteúdo adequadamente". Com advento do estudo hemodinâmico invasivo, Braunwald ${ }^{2}$ em 1992 publicou uma nova definição para IC que, acrescenta e destaca, o conceito das pressóes de enchimento e descreve a IC como "incapacidade do coração de bombear sangue de forma a atender às necessidades metabólicas tissulares, ou pode fazêlo somente com elevadas pressóes de enchimento". Esse conceito, embora útil do ponto de vista fisiopatológico, não é aplicado para maioria dos pacientes com IC e está presente somente nas formas terminais da doença.

John Cleland, importante cardiologista e líder global da pesquisa na IC, tem chamado atenção para dois aspectos, o primeiro, a importância da congestáo para definição IC e outro, a necessidade de uma definição universal. Além disso, a IC contemporânea tem destacado a busca pela etiologia e compreensão dos mecanismos fisiopatológicos, assim como a classificação em fenótipos clínicos. Esses pontos são fundamentais para definição e otimização terapêutica, que, em muitos casos, proporcionam mudança na história natural da doença com desfechos favoráveis. Carlos Chagas (Figura 1), ainda no início da década de 90, caracterizou a insuficiência cardíaca chagásica com a descrição de sinais e sintomas, como dispneia e edema de membros inferiores; dilatação das câmaras cardíacas, evidenciada pela ampliação marcada da silhueta cardíaca na radiografia de tórax. Descreveu, também, importantes descobertas sobre os aspectos histológicos de pacientes com cardiopatia crônica - infiltração de cé- 
lulas mononucleares e grave esclerose intersticial do miocárdio, atribuindo o quadro clínico a essas lesóes miocárdicas subjacentes.

Ou seja, a caracterização da IC como uma síndrome clínica associada a diferentes etiologias no contexto contemporâneo, tem a congestão como integrante fundamental nos sintomas e sinais clínicos, no impacto na qualidade de vida e necessidade de hospitalização e também no prognóstico. Portanto, mecanismos envolvidos na congestão têm se tornado uma importante meta diagnóstica e terapêutica. Diferentes métodos, invasivos e não invasivos - biomarcadores, ecodopppler, hemodinâmica invasiva, ultrassom pulmonar e bioimpedância torácica têm permitido a detecção precoce da congestão, até mesmo em sua fase pré sintomática.

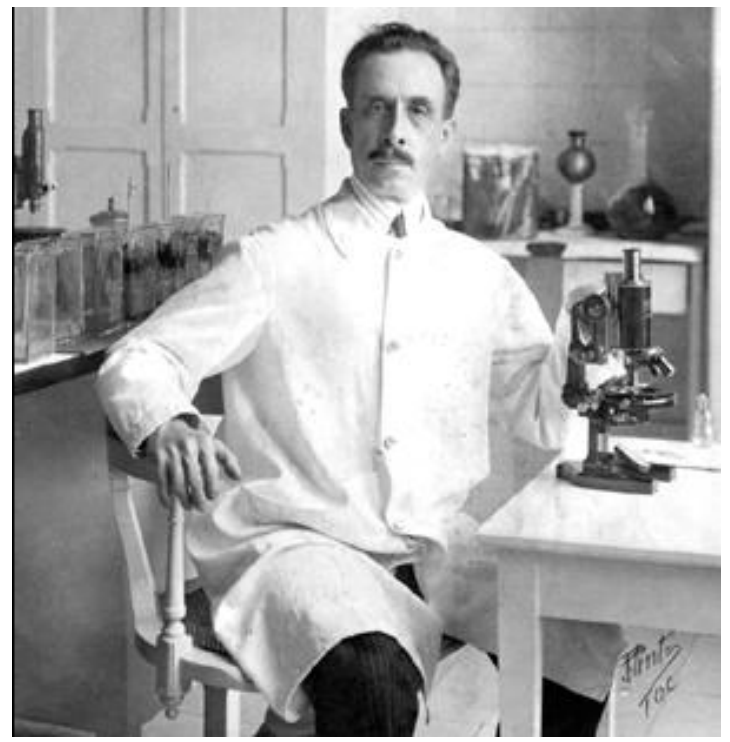

Figura 1: Carlos Chagas, em seu laboratório no Instituto Oswaldo Cruz mitido a detecção precoce da congestão, até mesmo em sua fase pré sintomática.

Em março de 2021 foi publicado, pioneira e simultaneamente, no Journal of Cardiac Failure e European Journal of Heart Failure, uma nova definição universal e classificação para insuficiência cardíaca (IC)3. Este documento foi elaborado pelas sociedades europeia, americana e japonesa de insuficiência cardíaca e apoiado pelas sociedades de IC canadense, indiana, chinesa, australiana e da Nova Zelândia. Os objetivos da publicação foram fornecer uma definiçáo universal, simples, mas abrangente, e que possa garantir uma padronizaçáo nas pesquisas clínicas, diretrizes, assistência e frente aos pacientes e formadores de políticas públicas; propor uma classificação revisada e baseada na fração de ejeção do ventrículo esquerdo (FEVE) a fim de guiar terapia de acordo com a categoria da IC e por fim, revisar os estágios da IC, visando prognóstico e com ênfase na prevenção.

As definiçóes são diferentes entre as diretrizes de diversas sociedades e divergem dos livros texto, trazem o conceito de sintomas e sinais associados a anormalidades hemodinâmicas e neuro-hormanais, que não são simples e facilmente mensuráveis. A nova definição é abrangente, permite unificar e facilitar o reconhecimento da IC e incorpora náo somente sinais e sintomas, mas também, marcadores objetivos de disfunção e causados por uma anormalidade 
cardíaca funcional ou estrutural e corroborada por elevação dos peptídeos natriuréticos (PNs) e/ou evidência de congestão pulmonar ou sistêmica (Figura 2). Os sinais e sintomas citados no documento foram expandidos e divididos em mais específicos e menos específicos e em típicos e atípicos, respectivamente (Quadro 1). Além disso, foi incorporado pela primeira vez valores de corte para PNs, assim como uma lista de situaçóes que poderiam influenciar seu valor. Isso foi um ponto importante, pois são biomarcadores com alto poder de aceitar ou rejeitar a síndrome e traz objetividade e facilidade para diagnóstico.
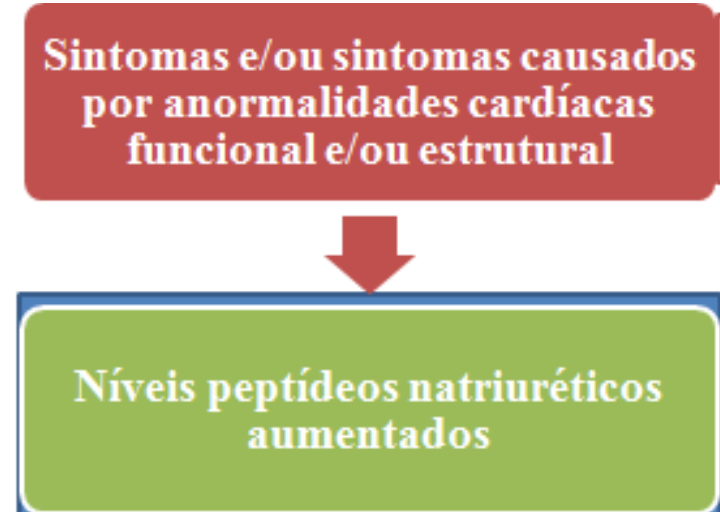

\section{ou}

\section{Evidência objetiva de congestão pulmonar ou sistêmica}

FEVE $<\mathbf{5 0} \%$, aumento anormal câmaras cardíacas, $\mathrm{E} / \mathrm{e}>15$, hipertrofia ventricular moderada/grave, lesão valvar obstrutiva ou regurgitante moderada/grave.
Ambulatorial:

$\mathrm{BNP}(\mathrm{pg} / \mathrm{ml}) \geq 35$ ou NT pro $\mathrm{BNP}(\mathrm{pg} / \mathrm{ml}) \geq 100$

Hospitalizado/ descompensado:

BNP $(\mathrm{pg} / \mathrm{ml}) \geq 100$ ou NT pro BNP $(\mathrm{pg} / \mathrm{ml}) \geq 300$

Figura 2: Nova definição universal de IC

A nova classificaçáo de acordo com a FEVE permite a construçáo de um fenótipo que orienta o tratamento. Destaca se a substituiçáo do termo "mid-range" por FE levemente reduzida uma vez que os dados atualmente disponíveis evidenciam benefício do bloqueio neurohomornal neste grupo de pacientes, semelhante aos re- sultados em pacientes com ICFER4. Outro ponto importante e que a nova definiçáo chama atenção é a trajetória da FEVE, pacientes com ICFER podem melhorar a FEVE com o manejo otimizado; assim como, pode ocorrer declínio acelerado da FE, sinalizando a necessidade de intensificação da terapia. 
- IC com FE reduzida: IC com FEVE $\leq$ $40 \%$.

- IC com FE levemente reduzida: IC com FEVE 41-49\%.

- IC com FE preservada: IC com FEVE $\geq 50 \%$.

- IC com FE melhorada: IC com FEVE basal $\leq 40 \%$, com aumento de 10 pontos FEVE basal e FEVE $\geq 40 \%$.

\begin{tabular}{|l|l|}
\hline \multicolumn{2}{|c|}{ Quadro 1: Sintomas e Sinais de IC } \\
\hline \multicolumn{2}{|c|}{ Sintomas } \\
\hline Típicos & Respicos \\
\hline Dispneia & Sensação ofegante \\
\hline Ortopneia & Plenitude pós prandial \\
\hline Dispneia paroxística noturna & Perda apetite \\
\hline Bendopneia & Declínio função cognitiva \\
\hline Fatiga / cansaço & Depressão \\
\hline $\begin{array}{l}\text { Tolerância reduzida ao } \\
\text { exercício }\end{array}$ & \\
\hline Incapacidade exercício & Tontura / síncope \\
\hline Edema & \\
\hline
\end{tabular}

\begin{tabular}{|l|l|}
\hline \multicolumn{2}{|c|}{ Sinais } \\
Mais específicos & \multicolumn{1}{|l|}{ Menos específicos } \\
\hline $\begin{array}{l}\text { Elevação pressão venosa } \\
\text { jugular }\end{array}$ & Edema periférico \\
\hline Terceira bulha cardíaca & Estertores pulmonares \\
\hline Ritmo galope & $\begin{array}{l}\text { Ganho de peso não } \\
\text { intencional (>2kg/sem) }\end{array}$ \\
\hline $\begin{array}{l}\text { Cardiomegalia, ictus } \\
\text { desviado lateralmente }\end{array}$ & $\begin{array}{l}\text { Perda de peso / caquexia (IC } \\
\text { avançada) }\end{array}$ \\
\hline Refluxo hepatojugular & Sopro cardíaco \\
\hline $\begin{array}{l}\text { Respiração de Cheyne- } \\
\text { Stokes (IC avanaçada) }\end{array}$ & $\begin{array}{l}\text { Taquicardia ; ritmo cardíaco } \\
\text { irregular }\end{array}$ \\
\hline & Taquipneia \\
\hline & Hepatomegalia / ascite \\
\hline & Extremidades frias \\
\hline & Oligúria \\
\hline & Pressão pulso estreita \\
\hline
\end{tabular}

Além disso, outro aspecto importante foi a revisão dos estágios da IC — risco para IC (estágio A) ; pré IC (estágio B) ; IC (estágio C) ; IC avançada (estágio D) (Figura 3). As denominaçóes para pacientes assintomáticos, em risco (A) e pré IC (B), terão um poder maior de convencimento sobre gravidade da doença, reforçando a prevenção e adesão à terapia, semelhante ao que acontece com o conceito de prémalignidade. Vale ressaltar, também, a adição de elevação dos biomarcadores (PNs ou troponina para aqueles expostos a agentes cardiotóxicos) como alternativa à alteração funcional ou estrutural no estágio $B$.

Algo inovador e também importante é a terminologia para compreensão da trajetória clínica do paciente: IC piorada (deteriorização dos sinais e sintomas apesar da progressão na terapia, requerendo hospitalizaçáo ou terapia avançada e venosa); IC persistente (ausência de melhora dos sintomas); IC em remissáo (resolução dos sinais e sintomas acompanhada de resolução de anormalidades cardíacas prévias). Resultados do estudo TRED-HF revelaram que $40 \%$ dos pacientes com cardiomiopatia dilatada que tiveram reversão do remodelamento e melhora dos sintomas com o tratamento ao suspenderem o esquema terapêutico apresentaram recidiva. Sugerindo remissão e não recuperação ${ }^{5}$. Outra substituição de destaque é "IC estável" por "IC persistente", importante para a compreensão do conceito 


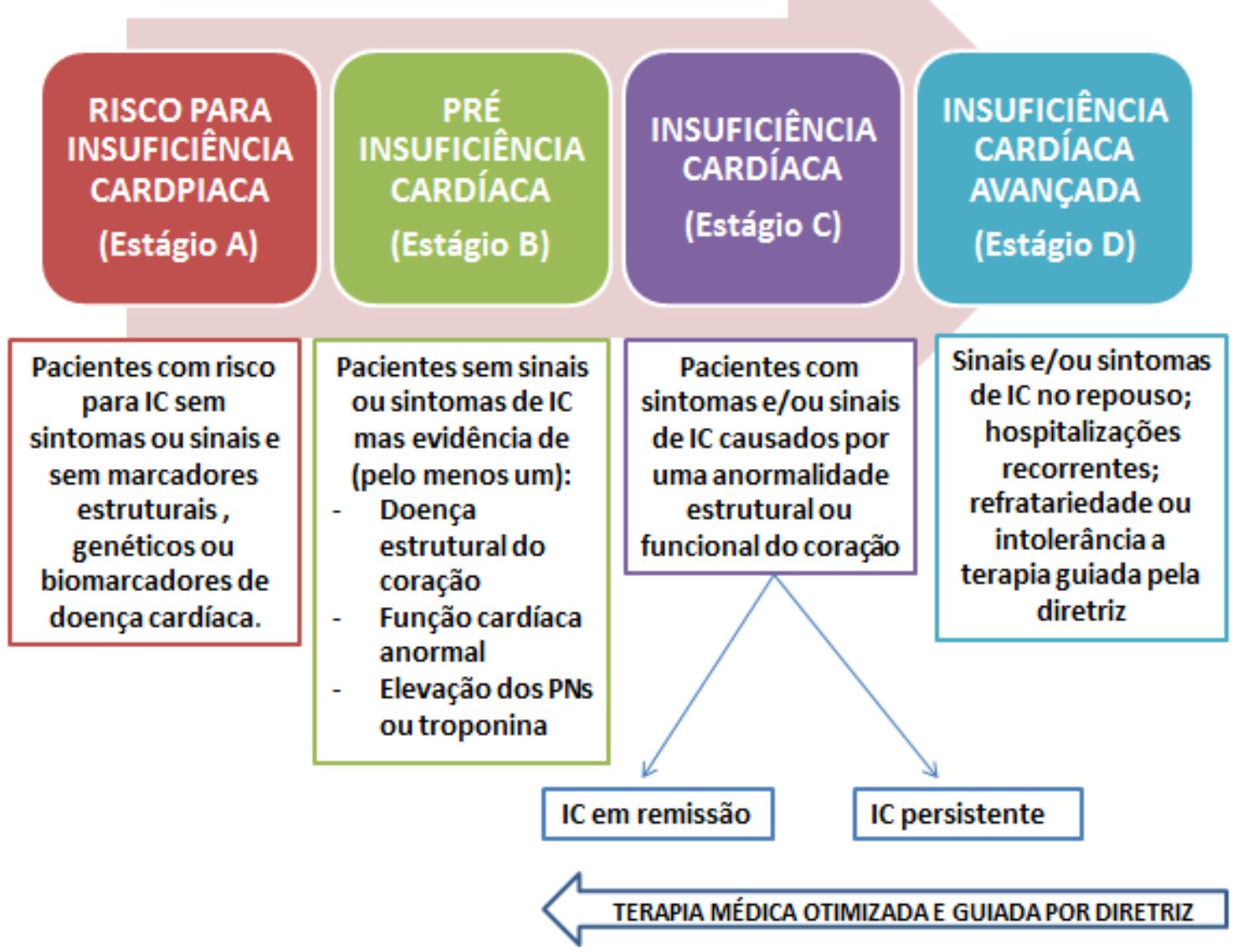

Figura 3: Estágios no desenvolvimento e progressão da IC.

terapia tempo sensível, evitando, portanto, a inércia terapêutica (Figura 2).

Essa nova definição e estadiamento da IC serão uma ferramenta útil para a padronização na assistência e na pesquisa da IC. Além disso, irão facilitar a abordagem e comunicação dos diferentes profissionais envolvidos com os pacientes com IC, cardio- logistas, especialistas em IC, enfermeiros, farmacêuticos, nutricionistas e demais membros da equipe multidisciplinar. Além de emergencistas, médicos clínicos, equipe da atenção primária e hospitalistas. Essa nova definição promoverá certamente uma profunda transformação e hemogeneização da classificação. E representara um marco, as- 
sim como a definição universal do infarto do miocárdio, hoje na sua quarta edição ${ }^{6}$.

\section{REFERÊNCIAS BIBLIOGRÁFICAS}

1. Lewis T. Diseases of the Heart. 1st ed. London, United Kingdom: MacMillian; 1933

2. Braunwald E. Heart Disease: A Textbook of Cardiovascular Medicine. 4th ed. Philadelphia, WB: Saunders; 1992.

3. Biykem Bozkurt, et al. Universal Definition and Classification of Heart Failure. Journal of Cardiac Failure 2021; 27(4): 387-413.

4. Marcondes-Braga FG, Moura LAZ, Issa VS, Vieira JL, Rohde LE, Simões MV, et al. Atualização de Tópicos Emergentes da Diretriz de Insuficiência Cardíaca - 2021. Arq Bras Cardiol. 2021; [online].ahead print, PP.0-0.

5. Halliday BP, Wassall R, Lota AS, Khalique Z, Gregson J, Newsome S, et al. Withdrawal of pharmacological treatment for heart failure in patients with recovered dilated cardiomyopathy (TRED-HF): an open-label, pilot, randomised trial. Lancet 2019;393:61-73.

6. Kristian Thygesen, Joseph S Alpert, Allan S Jaffe, Bernard R Chaitman,
Jeroen J Bax, David A Morrow, Harvey D White. Fourth Universal Definition of Myocardial Infarction (2018). Journal of the American College of Cardiology 2018; 72 (18): 2231-2264. 